\title{
Numerical model analysis and experimental verification of a thermal regulation strategy for thermal vacuum test
}

\author{
W. J. Ding, W. Shan, Z. J. Wang \& M. Liu \\ Beijing Institute of Spacecraft Environment Engineering, China
}

\begin{abstract}
The thermal regulation of a shroud has been widely applied in the thermal vacuum testing of spacecraft. The shroud is flooded with fluid, where the temperature is controlled to a specified range, so that the temperature of the test specimen can be changed with the shroud and maintained at a certain value as designed. A numerical model of temperature in the thermal regulation system is established and the heat transfer among fluids, the shroud and the test specimen are analyzed. A combination of heat radiation, heat conduction, and heat convection are realized based on the fuzzy control theory. When a test prototype is built, the test results show that the temperature range of the shroud can be steadily controlled between $-75^{\circ} \mathrm{C}$ and $155^{\circ} \mathrm{C}$, and the temperature of the test specimen can be stably controlled at any value in the range of $-50^{\circ} \mathrm{C}-120^{\circ} \mathrm{C}$. The over control of temperature is less than $1^{\circ} \mathrm{C}$, and the temperature stability is less than $\pm 0.5^{\circ} \mathrm{C}$.
\end{abstract}

Keywords: shroud, numerical model, test specimen, thermal regulation.

\section{Introduction}

A cold and black environment, where space vehicles suffer in orbit, is simulated by a shroud system of space environment simulator in a thermal vacuum test. The thermal regulator system of shroud refers to utilizing an external temperature regulator to control fluid, which is flooded into the shroud within the designed temperature range. The temperature variation of the shroud realizes the temperature regulation function of the specimen. The principle is independent in a thermal vacuum test, without any electric heating devices [1-5].

The configuration of the thermal regulator system changes due to different objectives of thermal tests, which is determined by different temperature ranges 
and temperature circulations. Moreover, temperature regulating difficulties encountered during the thermal vacuum test differ from different temperature circulations of the test specimen.

The temperature range of the thermal vacuum test for parts is from $-40^{\circ} \mathrm{C}$ to $+60^{\circ} \mathrm{C}$, and the corresponding temperature range of the shroud is from $-70^{\circ} \mathrm{C}$ to $+80^{\circ} \mathrm{C}$. Three types of temperature regulating systems can cover a temperature range of $-80^{\circ} \mathrm{C}-+150^{\circ} \mathrm{C}$ in the thermal vacuum test, which are alcohol-united with an infrared system, a gaseous nitrogen regulating system, and a thermal fluid regulating system. Among these systems, the thermal fluid regulating system has the advantages of quick operation, large thermal capacity of bath oil, small lag of temperature, and real simulation of temperature variation. Moreover, bath oil exists in the fluid state all of the time. Hence, a thermal regulating system with bath oil as a secondary refrigerant can meet the requirements of the thermal vacuum testing of electric products, as discussed in this paper.

\section{Numerical model and computational simulation}

The thermal regulator shroud selects thermal fluid as the cold source, and picks an electric heater to act as the heating source. The shroud is thermally regulated by thermal fluid, which is circulated by a pump according to the requirement of the control software. There are many advantages of this working principle, such as its simple configuration, lower cost for purchasing equipment, cheaper maintenance fee, and higher stability of the second refrigerant, all of which assure a good temperature uniformity of the shroud. Given the restrictions of refrigerator power and the head of the circulating pump, a regulating system is commonly applied in a medium or small thermal vacuum facility $[6,7]$.

A diagram of the working principle is shown in figure 1.

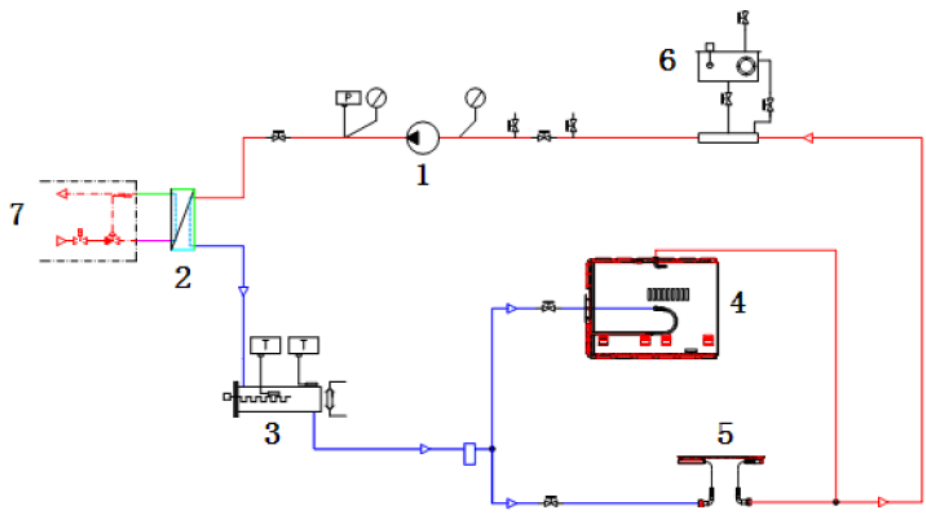

Figure 1: $\quad$ Temperature control system. $1=$ pump, $2=$ oil cooler, $3=$ oil heater, $4=\operatorname{Load}$ A, $5=$ load B, $6=$ oil expansion tank, $7=$ refrigeration system. 
The thermal regulation of the shroud is a closed loop control system, of which its objective is to maintain the temperature of the shroud precisely, as requested. The control feedback is the temperature of the specimen, which is measured and transmitted by the sensor and finally read by the computer. By comparing the updated temperature value of $\mathrm{PV}$ and the objective temperature SP using a computer, the difference e $=\mathrm{SP}-\mathrm{PV}$ is achieved. The output of the control software is decided by the calculation of the control strategy and the difference. By heating a second refrigerant to the specified temperature, the shroud increases to heat to the required temperature to realize the temperature regulation of the test specimen.

The cooling of the shroud is regulated through controlling the temperature of the refrigerant using the refrigerator. The start or stop of refrigerator combing with the action of a magnetic valve provides cold energy for the second refrigerant. The accurate control of the test specimen cannot be achieved without the help of an electric heater.

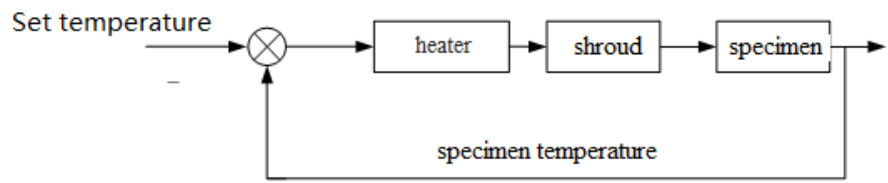

Figure 2: Control schematics of the temperature regulation system.

Fuzzy logic control, also known as fuzzy control, is an intelligent control method with flexibility and intelligence.

The simulation results are shown in figure 3 .

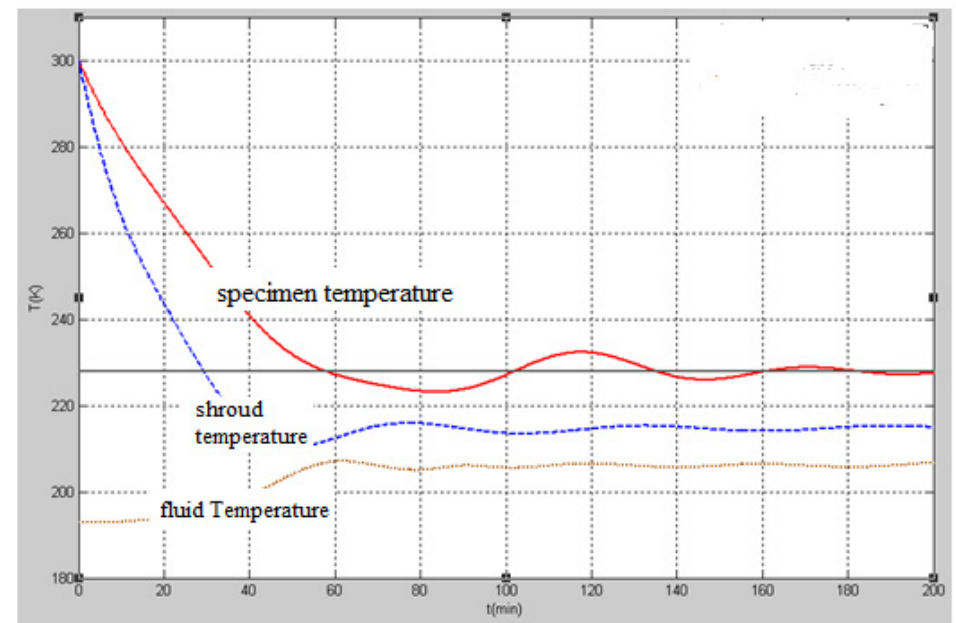

Figure 3: The simulation results. 
From the computation simulation above, the temperature of the test experiences a rapid decline at the beginning of regulation. When the temperature of the test specimen is close to the objective temperature, to prevent over regulating, the control parameters are adjusted to increase the temperature of the second refrigerant in order to decrease the decline velocity. When the system is stable, due to the stable error, there is a difference between the temperature of the test specimen and the objective temperature, which is in the permitted range. In the meantime, the results of the simulation show the temperature variation of the second refrigerant, with no apparent changes at the beginning, then a clear temperature increase, and almost reaching stability in the end. Because the temperature curve of the test specimen is eventually convergent, we can consider the second refrigerant temperature to be constant when the different $|\mathrm{e}|$ is controlled as per the required range.

\section{Test results and analysis}

\subsection{Test system}

The technical specifications of the shroud thermal regulating platform are as follows:

a) Horizontal vacuum chamber with door on one side, with the size of $\Phi 1200 \mathrm{~mm}$ $\times 1500 \mathrm{~mm}$;

b) Effective test space (shroud size): $\Phi 1000 \mathrm{~mm} \times 1200 \mathrm{~mm}$;

c) Support mechanism (cryopanel) for test specimen: size of $540 \mathrm{~mm} \times 800 \mathrm{~mm}$, which is mounted on the rail of the vacuum chamber;

d) Ultimate pressure with empty load: $<6.5 \times 10^{-5} \mathrm{~Pa}$.

The shroud thermal regulating platform is shown in figure 4 .

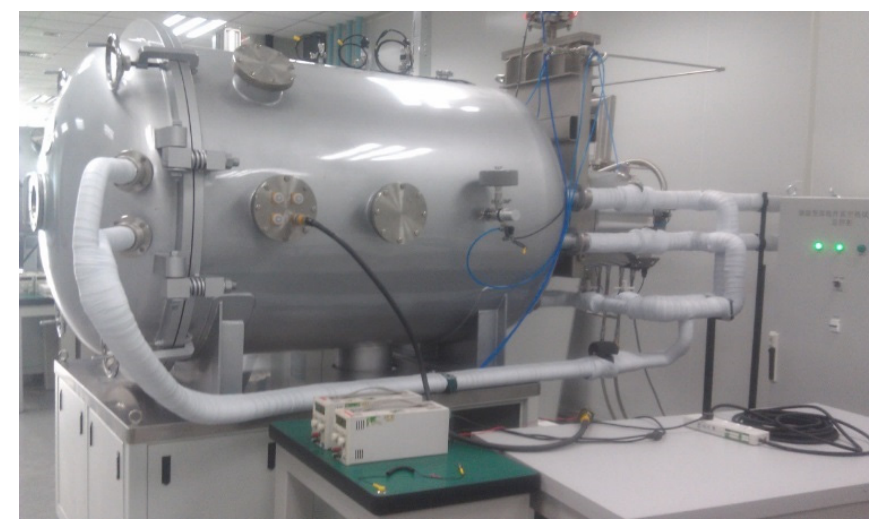

Figure 4: Test system.

The method of calculation for the temperature of the truss around the reflector and the staff between the net is similar to that of the cord, so does not need to be described again. 


\subsection{Test with an empty load}

Figure 5 shows the curves of the temperature variation of the shroud and the support mechanism with an empty load. Figure 6 shows the real temperature and the set temperature of the thermal fluid.

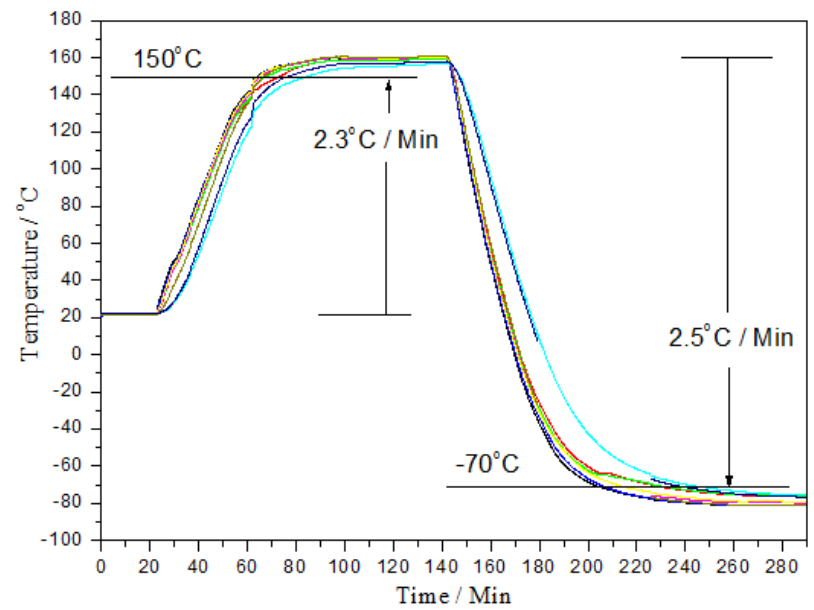

Figure 5: Temperature variation curves of the shroud and support mechanism.

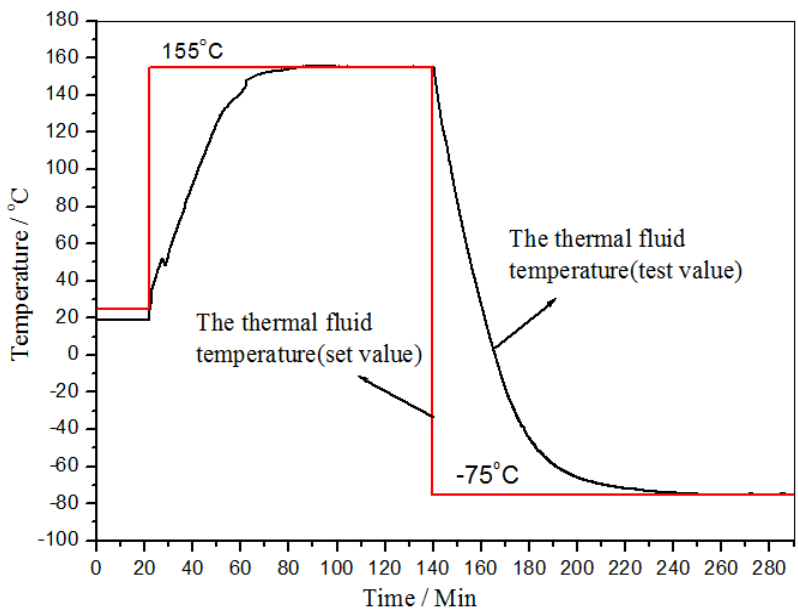

Figure 6: The curves of thermal fluid temperature variation.

From these two figures, it is clear that the shroud temperature with an empty load is from $-75^{\circ} \mathrm{C}$ to $155^{\circ} \mathrm{C}$ and the temperature uniformity is $\pm 2^{\circ} \mathrm{C}$. As for the cryopanel, the temperature uniformity is $\pm 1^{\circ} \mathrm{C}$. In addition, the temperature of the shroud and the cryopanel can be regulated and controlled at the intended value. 


\subsection{Test with test load}

To decide upon the temperature of the test specimen, as a controlled value, a stainless steel box that does not contain any inner heating source is used.

The test results are shown in figure 7.

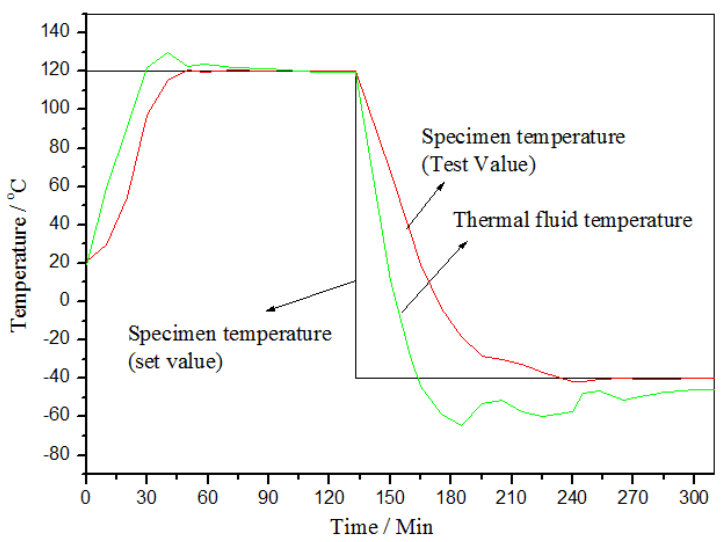

Figure 7: The test results (including the test load).

According to figure 7 , the thermal regulating system can realize the temperature control of the test specimen by regulating the temperature of the thermal fluid. The control accuracy of the temperature is $\pm 0.5^{\circ} \mathrm{C}$.

\section{Conclusion}

The thermal regulating system is analyzed, and a control model is developed, which is applied to the test facility. A test is conducted to realize the regulation of the shroud temperature from $-75^{\circ} \mathrm{C}$ to $155^{\circ} \mathrm{C}$ at a required value. High and low temperature circulation can be automatically realized with the temperature control accuracy of $\pm 0.5^{\circ} \mathrm{C}$.

\section{References}

[1] Wang Jun, Huang Bencheng, Wan Dacai. Technology of environment simulation [M]. Beijing: National Defense Industry Press, 1996: 490.

[2] Jiang Chuansheng, Wang Jun. Quantitative thermal design of heat sinks in large space simulation [J]. Journal of Beijing University of Aeronautics and Astronautics, 2001, 27(3): 305-308.

[3] Yu Tao, Liu Min, Zou Dingzhong. Thermal uniformity analysis for heat sink of space simulator [J]. Chinese Space Science and Technology, 2006, (6): $37-41$. 
[4] Yuan Xiugan, Liu Guoqing, Liu Min, et al. Simulation and analysis of flow and heat transfer uniformity in large liquid nitrogen heat sink [J]. Cryogenics, 2008, (2): 18-21.

[5] Zhang Lei, Liu Min, Liu Botao, Effects of Velocity and Inlet-outlet Form on Heat Transfer of Plate Heat Sink [J]. Spacecraft Environment Engineering, 2006, 23(4): 232-235.

[6] Yakhot V, Orzag S A. Renormalization group analysis of turbulence: basic theory [J]. J Scient. Comput., 1996, 1: 3-11.

[7] Yang Shiming, Tan Wenquan. Heat transfer [M]. Beijing: Higher Education Press, 1998: 134. 